KCL-TH-00-22

hep-th/0005135

October 31, 2018

\title{
On Harmonic Superspaces and Superconformal Fields in Four Dimensions
}

\author{
P. Heslop and P.S. Howe \\ Department of Mathematics \\ King's College, London
}

\begin{abstract}
Representations of four-dimensional superconformal groups on harmonic superfields are discussed. It is shown how various short representations can be obtained by parabolic induction. It is also shown that such short multiplets may admit several descriptions as superfields on different superspaces. In particular, this is the case for on-shell massless superfields. This allows a description of short representations as explicit products of fundamental fields. Superconformal transformations of analytic fields in real harmonic superspaces are given explicitly.
\end{abstract}




\section{Introduction}

The irreducible representations of the four-dimensional superconformal group $S U(2,2 \mid N)$ have been much studied in the literature [1]. The theory of such representations has assumed some significance recently in the light of the Maldacena conjecture [2 relating IIB supergravity and string theory on backgrounds with an $A d S_{5}$ factor to four-dimensional superconformal field theories on the boundary. A particularly important class of operators that can arise consists of those operators which correspond to short representations of the superconformal group since these are expected to be protected from quantum corrections and thus not acquire anomalous dimensions. (See, for example, [3, 4, 5]).

One way of constructing representations explicitly is the method of parabolic induction. In the context of superconformal groups this was discussed in some detail in [6], although a direct comparison with the more algebraic group-theoretic results of [1] was not made at the time. However, it was shown how certain ultra-short representations, which correspond to on-shell massless supermultiplets and which can be described by constrained superfields in ordinary $N$ extended Minkowski superspace [7, 8], can be described by "generalised chiral" or "Grassmann analytic" (G-analytic) superfields in harmonic superspaces [9]. A simple example of a composite operator - the $N=4$ supercurrent - was also constructed as the trace of the square of the $N=4$ Yang-Mills field-strength superfield. In [10] it was shown that there is in fact a series of such G-analytic superfields obtained by simply taking the trace of powers of the field strength, and it was conjectured that this circumstance might lead to restrictions on the correlations functions of such operators, an idea that has been exploited using both $N=4$ and $N=2$ superspaces in refs [11]. These composites all have maximum spin 2 and it was subsequently realised in [3] that the superconformal representations which are realised this way are dual to the bulk Kaluza-Klein representations which arise in the compactification of IIB supergravity on $A d S_{5} \times S^{5}$ [12]. In the case of an Abelian field strength this construction can easily be generalised to the case of the $D=6,(2,0)$ tensor multiplet and the $D=3, N=8$ scalar multiplet, these being the multiplets

which are relevant to M-theory compactified on $A d S_{4} \times S^{7}$ and $A d S_{7} \times S^{4}$ respectively [13] (for other discussions of $D=6,(2,0)$ representations see [14, 15, 16]).

In a recent series of papers short representations in $D=4$ [17, 18], $D=6$ [19] and $D=3$ [20] have been discussed in the context of harmonic superspaces which have the form of super Minkowski space times an internal coset space with isotropy group equal to the maximal torus in the internal symmetry group. As well as reproducing the KK multiplets the authors of these papers were able to obtain many other short representations by taking products of the underlying massless multiplets and then imposing harmonic analyticity on the outcome.

In the current paper we adopt a more general approach which makes use of all of the harmonic superspaces which are compatible with superconformal symmetry. We first discuss the short representations directly using the method of parabolic induction. To do this it is necessary to complexify spacetime and the superconformal group since the superspaces we are interested in are not cosets of the real superconformal group. One advantage of this approach is that superconformal symmetry is manifest throughout.

The short representations act on superfields which depend on fewer odd coordinates than are 
present in $N$-extended super Minkowski space; such G-analytic superfields obey constraints which generalise the constraints satisfied by chiral superfields. We show that each short representation generically admits realisations on different homogeneous superspaces which incorporate different numbers of G-analyticity constraints. Amongst these realisations there are those which are "maximally efficient" in that the maximum number of G-analyticity constraints compatible with the representation is explicitly incorporated. It will be seen that the number of G-analyticity constraints of a given realisation places constraints on the geometry of the internal manifold of the associated harmonic superspace. The fewer G-analyticity constraints there are (equivalently, the larger the number of odd coordinates of the superspace concerned) the larger the number of representations that will be compatible with it. Thus, G-analyticity of type $(1,1)$ is compatible with all representations of series C while G-analyticity of type $(1,0)$ (or $(0,1)$ ) is compatible with all short representations (see, for example, [5, 17] for discussions of these representations).

After a brief review of homogeneous superspaces of the superconformal group and parabolic induction, we discuss the short representations in detail in section 3 . In section 4 , we discuss on-shell massless supermultiplets from this viewpoint; these are special superconformal fields in that they carry ultra-short representations. Each of these massless supermultiplets can be realised in many different ways in general and this allows us to construct general representations out of them by taking products on appropriate superspaces. This approach has the virtue of not requiring the projection step of [17, 18] - the product superfields are naturally superconformal fields on the appropriate superspace. In particular, in non-Abelian $N=4$ supersymmetric Yang-Mills theory the single, double and triple trace gauge-invariant composite operators can be constructed straightforwardly as G-analytic and harmonic analytic superfields by making use of the three different realisations of the underlying field strength multiplet. Aspects of multiple trace operators, which are believed to correspond to multiparticle states in the AdS/CFT context, have been studied in [21] and in harmonic superspace in 17].

The paper concludes in section 5 with an explicit discussion of superconformal transformations acting on shortened supermultiplets defined as analytic superfields on real harmonic superspaces.

\section{Preliminaries}

\subsection{Harmonic superspace}

Harmonic superspaces are usually taken to be superspaces of the form $M \times F$ where $M$ is super Minkowski space and $F=H \backslash G$ is a coset space of the internal symmetry group $G$ with isotropy group $H$ [22, 23, 24, 6, 9]. Usually $F$ is a compact, complex manifold; in $N$-extended supersymmetry in four dimensions it can be taken to be of the form $\mathbb{F}_{\mathbf{k}}(N)=$ $S\left(U\left(k_{1}\right) \times U\left(k_{2}-k_{1}\right) \ldots U\left(N-k_{\ell}\right)\right) \backslash S U(N)$ where $\mathbf{k}:=\left(k_{1}, \ldots k_{\ell}\right)$ is a sequence of positive integers with $k_{1}<k_{2} \ldots k_{\ell}<N$ [25, 6, 9]. The space $\mathbb{F}_{\mathbf{k}}(N)$ is a flag manifold: a point in $\mathbb{F}_{\mathbf{k}}(N)$

is a flag in $\mathbb{C}^{N}$, i.e. a sequence of subspaces $\left\{V_{i}\right\}$, $\operatorname{dim} V_{i}=k_{i}$, such that $V_{1} \subset V_{2} \ldots V_{\ell} \subset \mathbb{C}^{N}$. Special cases of flag manifolds include projective spaces and Grassmannians. A complete flag is one with $\mathbf{k}=(1,2,3, \ldots N-1)$. The space of complete flags, which we shall denote $\mathbb{F}_{B}$, is 
$U(1)^{N-1} \backslash S U(N)$; it can also be thought of as the coset space $B \backslash S L(N, \mathbb{C})$ where $B$ is the Borel subgroup of $S L(N)$.

In the GIKOS formalism [22] one works with fields defined on $M \times G$ which are equivariant with respect to $H$. That is, one has fields $A(z, u), z \in M, u \in G$ which take their values in some representation space of $H$ and which satisfy $A(z, h u)=R(h) A(z, u), R$ being the representation of $H$ in question. Such fields are equivalent to sections of vector bundles over harmonic superspace. In index notation we write $u_{I}{ }^{i}$ where the upper case index indicates a representation of $H$ and the lower case index the defining representation of $G$, and where both indices run from 1 to $N$. The coordinates of Minkowski superspace are denoted by $\left(x^{\alpha \dot{\alpha}}, \theta^{\alpha i}, \bar{\theta}_{i}^{\dot{\alpha}}\right)$ where $\alpha$ and $\dot{\alpha}$ are two-component spinor indices. The covariant derivatives $\left(\partial_{\alpha \dot{\alpha}}, D_{\alpha i}, \bar{D}_{\dot{\alpha}}^{i}\right)$ satisfy

$$
\left[D_{\alpha i}, \bar{D}_{\dot{\alpha}}^{j}\right]=i \delta_{i}^{j} \partial_{\alpha \dot{\alpha}}
$$

with all other (anti)-commutators being zero. With the aid of $u$ we can convert $G$ indices to $H$ indices; this means that we can single out subsets of $D$ 's and $\bar{D}$ 's which anticommute with each other and which can therefore consistently be taken to annihilate superfields. Let us suppose that the index $I$ splits as follows under $H: I=\left(r, r^{\prime \prime}, r^{\prime}\right)$, where $r=1, \ldots p, r^{\prime \prime}=$ $p+1, \ldots N-q, r^{\prime}=N-q+1, \ldots N$, and where $(p+q) \leq N$, then we can have superfields $A$ which obey the constraints

$$
D_{\alpha r} A=\bar{D}_{\dot{\alpha}}^{r^{\prime}} A=0
$$

where

$$
D_{\alpha r}:=u_{r}^{i} D_{\alpha i} ; \quad \bar{D}_{\dot{\alpha}}^{r^{\prime}}=\bar{D}_{\dot{\alpha}}^{i}\left(u^{-1}\right)_{i}^{r^{\prime}}
$$

Constraints such as these are called Grassmann analyticity (G-analyticity) conditions following [26]; they generalise the notion of chirality which was first introduced in the context of $N=1$ supersymmetry in [27]. We shall refer to the G-analyticity conditions of equation (2) as being of type $(p, q)$. The space of $\mathrm{G}$-analytic structures of type $(p, q)$ on $N$-extended Minkowski superspace (in four dimensions) is the flag manifold $\mathbb{F}_{p, N-q}(N)=S(U(p) \times U(N-(p+q)) \times$ $U(q)) \backslash S U(N)$, and the harmonic superspace with this internal coset is referred to as $(N, p, q)$ harmonic superspace. Later on we shall make a refinement of this definition. The derivatives on $G$ can be taken to be the right-invariant vector fields, $D_{I}^{J}, D_{I}^{I}=0$. They act as follows:

$$
D_{I}^{J} u_{K}^{k}=\delta_{K}^{J} u_{I}^{k}-\frac{1}{N} \delta_{I}^{J} u_{K}^{k}
$$

and they obey the reality condition

$$
\bar{D}_{I}^{J}=-D_{I}^{J}
$$


These derivatives divide into three sets: the set $\left\{D_{r} s^{\prime}, D_{r} s^{\prime \prime}, D_{r^{\prime \prime}} s^{\prime}\right\}$, corresponding to the $\bar{\partial}$ operator on the complex coset space (i.e. holomorphic functions are annihilated by $\bar{\partial}$ ), the complex conjugate set $\left\{D_{r^{\prime}} s, D_{r^{\prime \prime}} s, D_{r^{\prime}} s^{\prime \prime}\right\}$ which corresponds to the $\partial$ operator, and the set $\left\{D_{r}{ }^{s}, D_{r^{\prime}} s^{\prime}, D_{r^{\prime \prime}} s^{\prime \prime}\right\}$ which corresponds to the isotropy algebra. As well as imposing G-analyticity we can impose harmonic analyticity (H-analyticity) by demanding that a superfield be annihilated by the first of these sets of derivatives. Indeed, since the algebra of all of these operators and the spinorial derivatives defining G-analyticity closes, it is consistent to impose both Ganalyticity and H-analyticity on the same superfield. Such superfields will be referred to as CR-analytic, or simply analytic. It was observed in 28] that analyticity conditions such as these define a generalisation of a complex structure, known as a CR structure, on harmonic superspace. In this language, a G-analytic structure can be considered to be a CR-structure which is purely odd.

\subsection{Harmonic conjugation}

It was noted in the first paper on harmonic superspace 22] that it is necessary to use a modified conjugation in order to handle real representations of $S U(2)$ in a straightforward manner. This conjugation combines the antipodal map on the internal manifold $\mathbb{C} P^{1}$ with ordinary complex conjugation. This was generalised to $(N, p, q)=(3,1,1)$ in [23] and to any $(N, p, p)$ space

in [9]. In a general $(N, p, q)$ superspace, with internal manifold $\mathbb{F}_{p, N-q}(N)$ the analogue of the antipodal map becomes a map from $\mathbb{F}_{p, N-q}(N)$ to $\mathbb{F}_{q, N-p}(N)$; if we combine this transformation with complex conjugation we obtain a map which maps the CR-structure on $(N, p, q)$ harmonic superspace to the $\mathrm{CR}$ structure on $(N, q, p)$ superspace. We shall refer to this as harmonic conjugation.

Working on $M \times S U(N)$ we write $u_{I}^{i}$ as before as $\left(u_{r}^{i},{u_{r^{\prime \prime}}}^{i}, u_{r^{\prime}}{ }^{i}\right)$ with $r=1, \ldots p$ and $r^{\prime}=$ $N-q+1, \ldots N$. This is the appropriate splitting of $I$ for $(N, p, q)$ superspace. We denote the splitting relevant to $(N, q, p)$ by $I=\left(R, R^{\prime \prime}, R^{\prime}\right)$ with $R=1, \ldots q, R^{\prime}=N-p+1, \ldots N$. The mapping we start with is given by

$$
u \mapsto k u
$$

where $k$ is the matrix

$$
k=\left(\begin{array}{ccc}
0 & 0 & -1_{q} \\
0 & 1_{r} & 0 \\
1_{p} & 0 & 0
\end{array}\right)
$$

It is easy to check that $k h k^{-1} \in S(U(q) \times U(N-(p+q)) \times U(p))$ provided that $h \in S(U(p) \times$ $U(N-(p+q)) \times U(q))$, so that this does indeed define a map from one coset space to the other, i.e. from $\mathbb{F}_{p, N-q}(N)$ to $\mathbb{F}_{q, N-p}(N)$. If we combine this with complex conjugation we find

$$
u_{r}^{i} \mapsto\left(u^{-1}\right)_{i}^{R^{\prime}}
$$




$$
\begin{aligned}
u_{r^{\prime \prime}}{ }^{i} & \mapsto\left(u^{-1}\right)_{i}^{R^{\prime \prime}} \\
u_{r^{\prime}}{ }^{i} & \mapsto-\left(u^{-1}\right)_{i}{ }^{R}
\end{aligned}
$$

For the inverses we have

$$
\begin{aligned}
\left(u^{-1}\right)_{i}{ }^{r} & \mapsto u_{R^{\prime}}{ }^{i} \\
\left(u^{-1}\right)_{i}{ }^{r^{\prime \prime}} & \mapsto u_{R^{\prime \prime}}{ }^{i} \\
\left(u^{-1}\right)_{i}{ }^{r^{\prime}} & \mapsto-u_{R}{ }^{i}
\end{aligned}
$$

We then find

$$
\begin{aligned}
D_{\alpha r} & \mapsto \bar{D}_{\dot{\alpha}}^{R^{\prime}} \\
\bar{D}_{\dot{\alpha}}^{r^{\prime}} & \mapsto-D_{\alpha R}
\end{aligned}
$$

while

$$
\begin{aligned}
D_{r} s^{\prime} & \mapsto D_{S} R^{\prime} \\
D_{r} s^{\prime \prime} & \mapsto-D_{S^{\prime \prime}} R^{\prime} \\
D_{r^{\prime \prime}} s^{\prime} & \mapsto D_{S} R^{\prime^{\prime \prime}}
\end{aligned}
$$

so that the derivatives of the $(N, p, q)$ CR-structure do indeed transform into those of the $(N, q, p)$ CR-structure, and therefore analytic fields on $(N, p, q)$ superspace transform into analytic fields on $(N, q, p)$ superspace.

\subsection{Complex superspaces}

It is the analytic superfields defined on harmonic superspaces of the above type, or related ones, that we shall be interested in for the description of short representations of the superconformal group. However, from a group-theoretical point of view, it is convenient to take a slightly different approach to harmonic superspaces, namely as coset spaces of the superconformal group. A slight technical problem presents itself here, because the spaces we are ultimately interested in, i.e. the so-called analytic superspaces where the G-analyticity constraints are automatically solved, are not coset spaces of the real superconformal group $S U(2,2 \mid N)$; we can circumvent this problem by complexifying both the group and spacetime. The fields we are interested in will then be holomorphic fields defined on coset spaces of the complexified superconformal group $S L(4 \mid N)$ (we omit the $\mathbb{C}$ which is always understood). The isotropy groups will be parabolic subgroups, which means that the superspaces themselves will be flag supermanifolds, that is, spaces whose

points correspond to nested sequences of sub-supervector spaces of $\mathbb{C}^{4 \mid N}$. The internal parts of these spaces will, however, be the same as in the real case above, the only difference being 
that we treat them exclusively as complex manifolds. When we restrict spacetime and the odd coordinates to be real we recover analytic fields of the type we have discussed above on $(N, p, q)$ harmonic superspaces. The virtue of this approach is that superconformal covariance is manifest.

We begin by considering complexified Minkowski space which can be viewed as an open subset of the coset space $P \backslash S L(4), S L(4)$ being the complexified conformal group and $P$ the subgroup of matrices of the following shape:

$$
\left(\begin{array}{llll}
\bullet & \bullet & & \\
\bullet & \bullet & & \\
\bullet & \bullet & \bullet & \bullet \\
\bullet & \bullet & \bullet & \bullet
\end{array}\right)
$$

where the bullets denote elements which do not have to be zero. The blank region can be thought of as corresponding to spacetime. Indeed, we can choose a coset representative of the form

$$
M \ni x \mapsto s(x)=\left(\begin{array}{cc}
1_{2} & x \\
0_{2} & 1_{2}
\end{array}\right)
$$

where each entry is a two-by-two matrix. From this one can easily work out that the transformation of $x$ under the conformal group is the usual one by using standard homogeneous space techniques.

Complexified super Minkowski space has the form $P \backslash S L(4 \mid N)$ where $P$ consists of matrices of the form

$$
\left(\begin{array}{cccc|ccc}
\bullet & \bullet & & & & & \\
\bullet & \bullet & & & & & \\
\bullet & \bullet & \bullet & \bullet & \bullet & \cdot & \bullet \\
\bullet & \bullet & \bullet & \bullet & \bullet & \cdot & \bullet \\
\hline \bullet & \bullet & & & \bullet & \cdot & \bullet \\
\bullet & \bullet & & & \cdot & \cdot & \cdot \\
\bullet & \bullet & & & \bullet & \cdot & \bullet
\end{array}\right)
$$

The standard coset representative is

$$
M \ni z \mapsto s(z)=\left(\begin{array}{cc|c}
1_{2} & x & \theta \\
0 & 1_{2} & 0 \\
\hline 0 & \varphi & 1_{N}
\end{array}\right)
$$

where $\varphi$ denotes the $N$ dotted spinorial coordinates which become the complex conjugates of the $\theta$ 's in the real case.

Complexified $(N, p, q)$ harmonic superspace is given by the following subgroup 


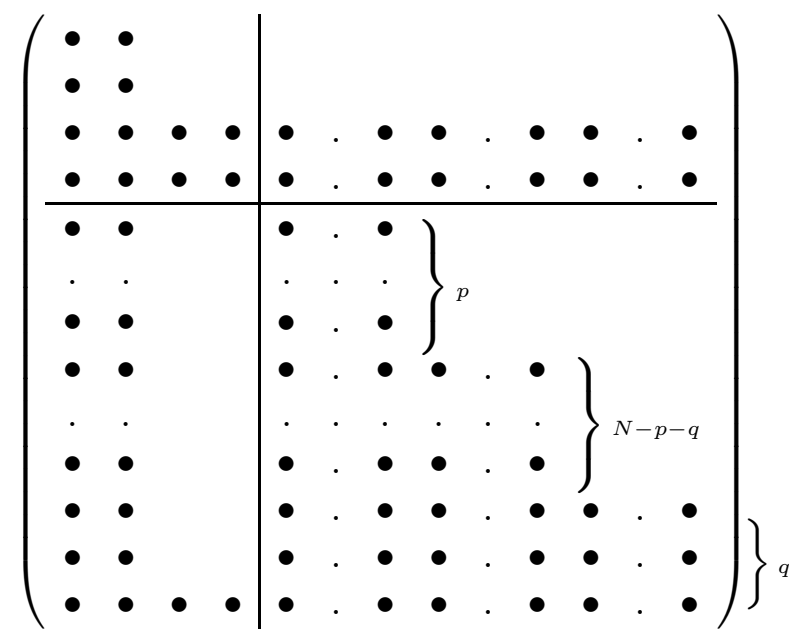

Locally, this space has the form of complex super Minkowski space times the internal flag manifold $\mathbb{F}_{p, N-q}(N)$. The related $(N, p, q)$ analytic superspace has the same body but fewer odd coordinates; the relevant subgroup is

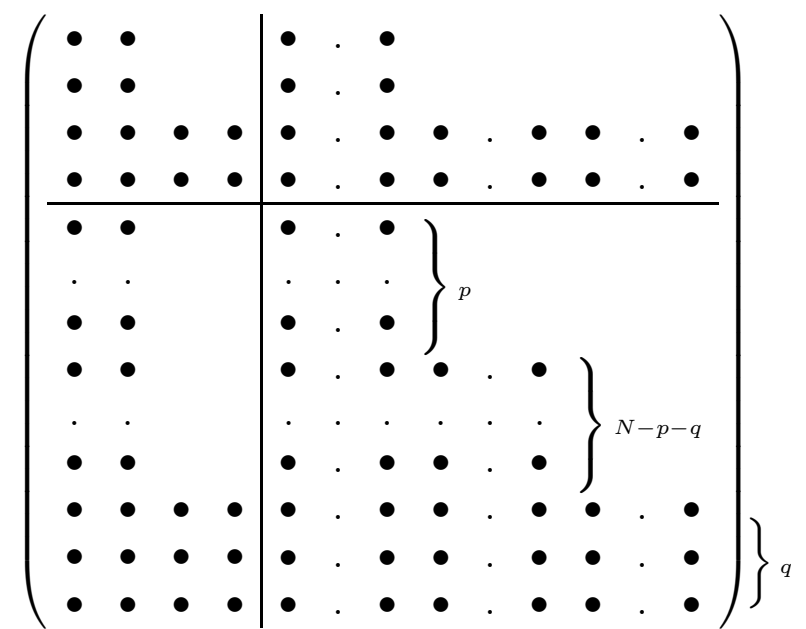

A CR-analytic field defined on real $(N, p, q)$ harmonic superspace, when continued to complexified superspace, will be a holomorphic field defined on the above $(N, p, q)$ analytic superspace.

\subsection{Parabolic induction}

If $G$ is a Lie group and $H$ a subgroup, representations of $G$ can be induced from representations of $H$ by considering $H$-vector bundles over $H \backslash G$. Sections of such bundles are, as we mentioned

previously, equivalent to equivariant maps $F: G \rightarrow V$, where $V$ is the representation space of $H$ with representation $R$, say. Thus we have (for $u \in G$ ) 


$$
F(h u)=R(h) F(u)
$$

The induced representation itself is given by $F \mapsto g \cdot F, g \in G$ where

$$
(g \cdot F)(u)=F(u g)
$$

It is easy to see that this does define a (left) representation of $G$ carried by the space of sections of the vector bundle in question. Parabolic induction refers to the case where the subgroup is parabolic. In this case, if the representation $R$ is irreducible then the representation of $G$ constructed in this manner is also irreducible. (For a detailed discussion of parabolic subgroups and flag manifolds in the context of twistor theory see [29].)

Let $\mathrm{G}$ be a complex, simple Lie group and let $\mathfrak{g}$ be its Lie algebra. A Borel subalgebra $\mathfrak{b}$ is a maximal solvable subalgebra of $\mathfrak{g}$ and a parabolic subalgebra is one which contains a Borel subalgebra. For the case of $\mathfrak{s l}(N)$ we may take the Borel subalgebra to be the algebra of all lower triangular matrices (with non-zero entries on the diagonal allowed), and a parabolic subalgebra $\mathfrak{p}$ is one which is block lower triangular. Thus it consists of matrices of the form:

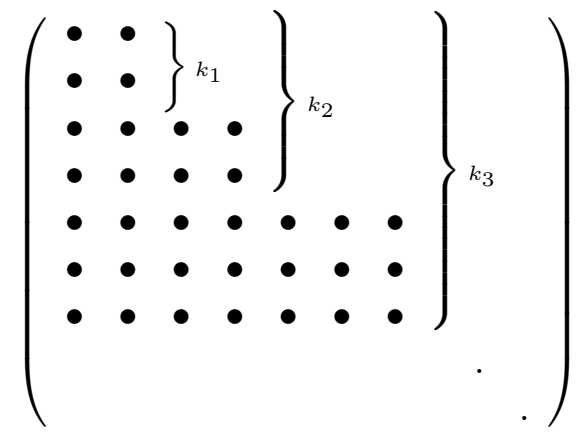

where, if the squares have sides $k_{1}, k_{2}, \ldots k_{\ell}$, the parabolic specifies the flag manifold $\mathbb{F}_{\mathbf{k}}(N)$, where $\mathbf{k}=\left(k_{1}, k_{2}, \ldots k_{\ell}\right)$. The parabolic $\mathfrak{p}$ can be represented by placing a cross on each of the nodes $k_{1}, k_{2}, \ldots k_{\ell}$ of the Dynkin diagram for $\mathfrak{s l}(N)$. For example, the flag manifold $\mathbb{F}_{1,3}(4)$, as well as the corresponding parabolic subalgebra, is represented by the $\mathfrak{s l}(4)$ Dynkin diagram $\ltimes \bullet \times$. Thus we have two constructions of flag manifolds - as homogeneous spaces of $S U(N)$ with isotropy groups of the type discussed previously, which makes it clear that they are compact, and as homogeneous spaces of $S L(N)$ defined by parabolic isotropy groups, which makes it clear that they are complex manifolds. As we mentioned previously, the Borel subgroup defines the space of flags of type $\mathbf{k}=(1,2,3, \ldots(N-1))$; it is the same space as the homogeneous space of $S U(N)$ which has the maximal torus as its isotropy group. 
The sub-algebra of $\mathfrak{p}$ consisting of the blocks along the diagonal is called the Levi sub-algebra $\mathfrak{l}$ and we have $\mathfrak{p}=\mathfrak{l} \oplus \mathfrak{u}$ where $\mathfrak{u}$ consists of the remaining matrices; clearly $\mathfrak{u}$ is nilpotent, $\mathfrak{u}_{k}=0$ for some $k$ where $\mathfrak{u}_{k}$ is defined iteratively by $\mathfrak{u}_{k}=\left[\mathfrak{u}_{k-1}, \mathfrak{u}_{k-1}\right] ; \mathfrak{u}_{0}=\mathfrak{u}$. For a general flag manifold $\mathbb{F}_{\mathbf{k}}(N)$, the Levi subalgebra is

$$
\mathfrak{l}=\mathfrak{s l}\left(k_{1}\right) \oplus \mathfrak{s l}\left(k_{2}-k_{1}\right) \oplus \ldots \oplus \mathfrak{s l}\left(N-k_{\ell}\right) \oplus \mathbb{C}^{\ell}
$$

The finite-dimensional irreducible representations of $\mathfrak{s l}(N)$ are specified by highest weights $\lambda$. These representations can alternatively be constructed by parabolic induction. In fact they act on holomorphic sections of homogeneous vector bundles over the flag manifolds $P \backslash S L(N)$. This procedure can be described explicitly. Choose a representation with highest weight $\lambda$ and fix a parabolic $\mathfrak{p}$. Any weight for $\mathfrak{s l}(N)$ is also a weight for any $\mathfrak{p}$ and so highest weights determine irreducible representations of $\mathfrak{p}$ in some vector space $V_{\lambda}$. In fact, since $\mathfrak{u}$ is nilpotent, it acts trivially on such a representation, so that we actually have a representation of the Levi subalgebra of $\mathfrak{p}$ on $V_{\lambda}$. We then construct the vector bundle $\mathcal{V}_{\lambda}$ over $P \backslash S L(N)$, i.e. the bundle whose standard fibre is the $P$-representation space $V_{\lambda}$. A simple application of the Bott-BorelWeil theorem gives the desired result: the space of holomorphic sections of $\mathcal{V}_{\lambda}$ is isomorphic to the representation space of $\mathfrak{s l}(N)$ determined by the highest weight $\lambda$. Note that a given representation can be presented in this fashion in as many different ways as there are parabolic subalgebras of $\mathfrak{s l}(N)$. ( A discussion of this theorem in the context of twistor theory is given in [29]).

This discussion can be made more concrete as follows: a holomorphic section, $f$, of the vector bundle $\mathcal{V}_{\lambda}$ is the same thing as a holomorphic map, $F$, from $S L(N)$ to the representation space $V$ which is equivariant with respect to the subgroup $P$ :

$$
F(p u)=R(p) F(u)
$$

where $R$ denotes the representation in question (and is only non-trivial for the Levi subgroup $L$ ), and where $p \in P, u \in S L(N)$. If the representation in question has Dynkin labels $\left(a_{1}, \ldots, a_{N-1}\right)$, it can be represented by a Young tableau with $m_{l}$ boxes in the $l$ th row, $l=1, \ldots, N-1$ with

$$
m_{l}=\sum_{k=l}^{N-1} a_{k}
$$

Denote an element of $S L(N)$ by $u_{I}^{i}$. The index $I$ splits under the Levi sub-algebra as $I=$ $\left(r_{1}, r_{2}, \ldots r_{\ell+1}\right)$, where $r_{i}$ runs from $k_{i-1}$ to $k_{i}$, with $k_{0}=1$ and $k_{\ell+1}=N$. The desired holomorphic section corresponding to the representation with Dynkin labels is schematically of the form

$$
F(u) \sim\left(u_{1}\right)^{m_{1}+m_{2}+\ldots m_{k_{1}}}\left(u_{2}\right)^{m_{k_{1}+1}+\ldots m_{k_{2}}} \ldots\left(u_{\ell+1}\right)^{m_{k_{\ell}} \ldots m_{N-1}} \hat{F}
$$

where $u_{1}$ denotes the $k_{1} \times N$ matrix $u_{r_{1}}{ }^{i}$, etc. and where $\hat{F}$ on the right-hand side denotes the $\mathrm{SL}(\mathrm{N})$ tensor with a total of $m=\sum_{k=1}^{N-1} k a_{k}$ (subscript) indices. 


\subsection{Flag supermanifolds}

A super-flag in a supervector space $\mathbb{C}^{m \mid n}$ is a nested sequence of sub-supervector spaces $V_{1} \subset$ $V_{2} \ldots V_{\ell} \subset \mathbb{C}^{m \mid n}$ where the super-dimension of $V_{i}$ is $K_{i}=\left(k_{i} \mid \kappa_{i}\right), k_{i}\left(\kappa_{i}\right)$ being the dimensions of the even (odd) subspaces of $V_{i}$ respectively, with $K_{i}<K_{j}$ for $i<j$. This notation means that $k_{i} \leq k_{j}, \kappa_{i} \leq \kappa_{j}$, but with equality for both even and odd indices prohibited. The space of such super-flags will be denoted $\mathbb{F}_{\mathbf{K}}(m \mid n)$, where $\mathbf{K}=\left(K_{1}, \ldots K_{\ell}\right)$ [30]. In the present paper we are interested in super-flags in $\mathbb{C}^{4 \mid N}$, and they are determined by various parabolic subgroups of $S L(4 \mid N)$. In general, there are three main classes of such spaces according to whether the body is spacetime times an internal flag manifold, a twistor space (in the usual sense), or a combination of both, i.e. a twistor space times an internal flag manifold. We shall be interested in the first of these types of space since they correspond to (complexified) harmonic superspaces. Moreover, we shall be interested in superspaces which correspond to G-analyticity of type $(p, q)$. In the complex setting this means that we want to restrict our attention to spaces which have $(N-p)$ two-component undotted spinor coordinates and $(N-q)$ two-component dotted spinor coordinates. The relevant flag supermanifolds are thus given by $\mathbf{K}=\left(2|p, 2| p+k_{1}, \ldots 2\left|p+k_{\ell}, 2\right|(N-q)\right)$. The body of such a supermanifold is locally the product of spacetime with the internal flag manifold $\mathbb{F}_{p, p+k_{1}, \ldots, p+k_{\ell}, N-q}(N)$. Such supermanifolds are generalisations of the $(N, p, q)$ analytic superspaces introduced in [9]. The latter are the flag supermanifolds $\mathbb{F}_{2|p, 2| N-q}(4 \mid N)$; they are the spaces with the smallest internal manifolds for a given choice of $(p, q)$. The $(N, p, q)$ harmonic superspaces have internal flag manifolds $\mathbb{F}_{p, N-q}(N)$ with associated Levi sub-algebras $\mathfrak{s l}(p) \oplus \mathfrak{s l}(q) \oplus \mathfrak{s l}(r) \oplus \mathbb{C}^{2}$, where $r=N-(p+q)$. The generalised $(N, p, q)$ spaces differ in that a non-trivial internal space is allowed corresponding to the central $r \times r$ sector of the internal symmetry group.

An important consequence of these definitions is that not all possible internal flag manifolds are compatible with a given $(p, q)$ G-analyticity and superconformal symmetry. For example, if either $p$ or $q$, or both, is bigger than 1 , then the internal parabolic cannot be taken to be the Borel subalgebra. T This may be clearly seen from the following pictorial representation of the parabolic subalgebra of $\mathfrak{s l}(4 \mid N)$ for $(N, p, q)$ analytic superspace:

\footnotetext{
${ }^{1}$ This would seeem to contradict refs [17, 18]. However, the harmonic superfields for the underlying massless supermultiplets used by these authors satisfy further harmonic anti-analytic constraints which means that they actually live on $(N, p, q)$ spaces; in fact, they are the same fields as those of [9].
} 


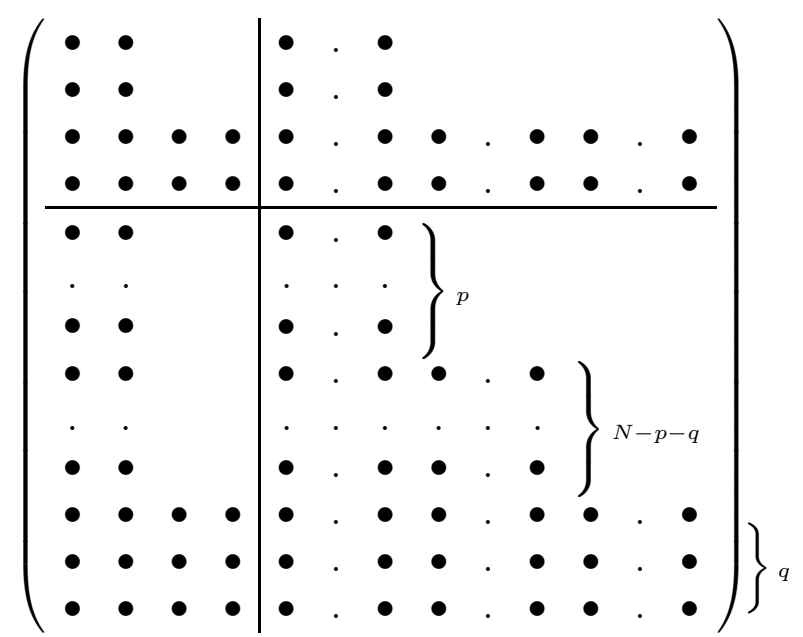

Multiplication of odd elements of the algebra (i.e. the bottom left times the top right) clearly generates the $p \times p$ and $q \times q$ parts of the internal algebra which must therefore be filled with bullets. The remaining central part of the algebra can be completed in many different ways so that an internal parabolic subalgebra is obtained; we have depicted the simplest example here corresponding to the flag space $\mathbb{F}_{p, N-q}(N)$.

The group $S L(4 \mid N)$ acts to the left on $\mathbb{C}^{4 \mid N}$ which may be considered as $N$-extended nonprojective super twistor space [31]. To exhibit the flag nature of the above superspaces more clearly it is convenient to make a change of basis of $\mathbb{C}^{4 \mid N}$. If a supertwistor $Z$ is written as

$$
Z_{A}=\left(\begin{array}{c}
z^{\alpha} \\
z_{\dot{\alpha}} \\
\zeta_{i}
\end{array}\right)
$$

then the required change of basis swaps $z_{\dot{\alpha}}$ and $\zeta_{i}$.

$$
Z_{A} \rightarrow\left(\begin{array}{c}
z^{\alpha} \\
\zeta_{i} \\
z_{\dot{\alpha}}
\end{array}\right)
$$

In this basis the above parabolic takes the following form: 


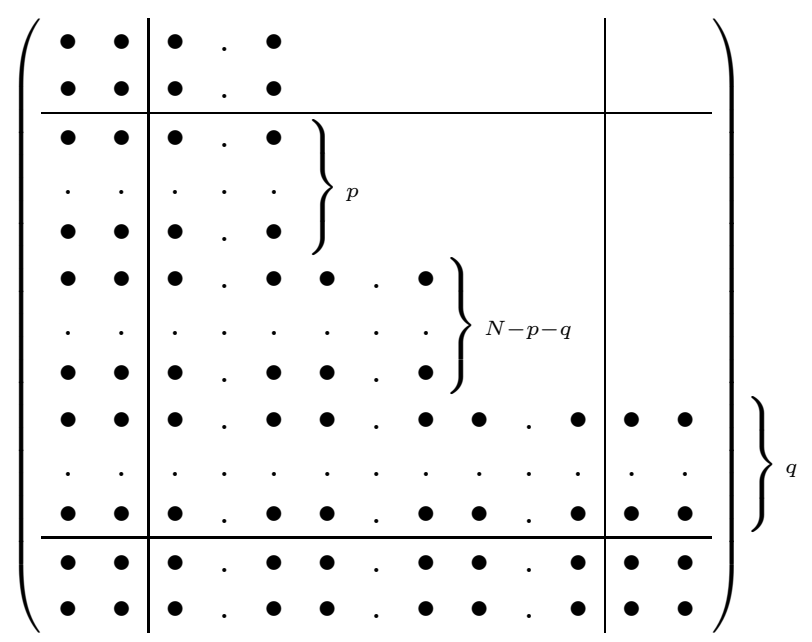

This brings it to block lower-triangular form as in the bosonic case. 2 The Levi subalgebra for this parabolic is

$$
\mathfrak{l}=\mathfrak{s}(\mathfrak{g l}(2 \mid p) \oplus \mathfrak{g l}(2 \mid q) \oplus \mathfrak{g l}(r))
$$

provided that $N \neq 4$. For non-exceptional cases, i.e. when neither $p$ nor $q$ equals 2 and when $r \neq 0$, this algebra is isomorphic to $\mathfrak{s l}(2 \mid p) \oplus \mathfrak{s l}(2 \mid q) \oplus \mathfrak{s l}(r) \oplus \mathbb{C}^{2}$. We shall discuss the exceptional cases in the next section in more detail. In the case of generalised $(N, p, q)$ spaces, the central $\mathfrak{s l}(r)$ summand is replaced by an appropriate parabolic subalgebra.

\section{Short representations}

We can apply the method of parabolic induction straightforwardly in the supersymmetric case to obtain representations of $S L(4 \mid N)$ as holomorphic sections of homogeneous vector bundles over flag supermanifolds defined by parabolic subgroups of the type we have discussed above. The short representations are characterised by being short multiplets and thus having lower component spins than unconstrained superfields on Minkowski superspace. Such representations act naturally on superfields defined on analytic superspaces since they have fewer odd coordinates than Minkowski superspace.

The superfields carrying the representations should transform under irreducible representations of Levi subalgebras of the form $\mathfrak{l}=\mathfrak{s}(\mathfrak{g l}(2 \mid p) \oplus \mathfrak{g l}(2 \mid q) \oplus \mathfrak{g l}(r))$; however, in order to ensure that the representations are indeed short these superfields must not carry any spacetime indices. They must therefore transform trivially under any supergroup factors of the Levi subgroup. In the generic case this means that they transform only under $\mathfrak{s l}(r) \oplus \mathbb{C}^{2}$, where we recall that $r=N-(p+q)$. Another point to be borne in mind is that the same representation can be

\footnotetext{
${ }^{2}$ In this basis the Borel subalgebra consists of the lower triangular matrices; note that it is possible to have inequivalent Borel subalgebras in the super case.
} 
realised on different superspaces corresponding to different parabolic subgroups; in particular, for a given representation there will be a class of "maximally efficient" realisations, by which we mean that the shortening is the largest possible, i.e. $p$ and $q$ take their largest possible values for the given representation. There may be several representations of this type due to the fact that one is free to choose the central factor of the internal parabolic in different ways.

In order to keep matters as simple as possible, we shall concentrate for the time being on $(N, p, q)$ superspaces which have internal flag manifolds $\mathbb{F}_{p, N-q}(N)$. The representations to be studied can then be represented by modified Dynkin diagrams of the following type:

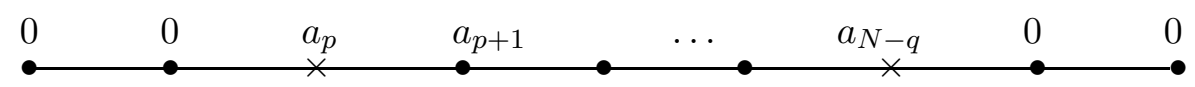

For the reasons discussed above the first $(p-1)$ and the last $(q-1)$ Dynkin labels must vanish, leaving $(r-1)$ labels to specify the representation of the central $\mathfrak{s l}(r)$ and two further labels which specify the charges. The Young tableau for this representation is

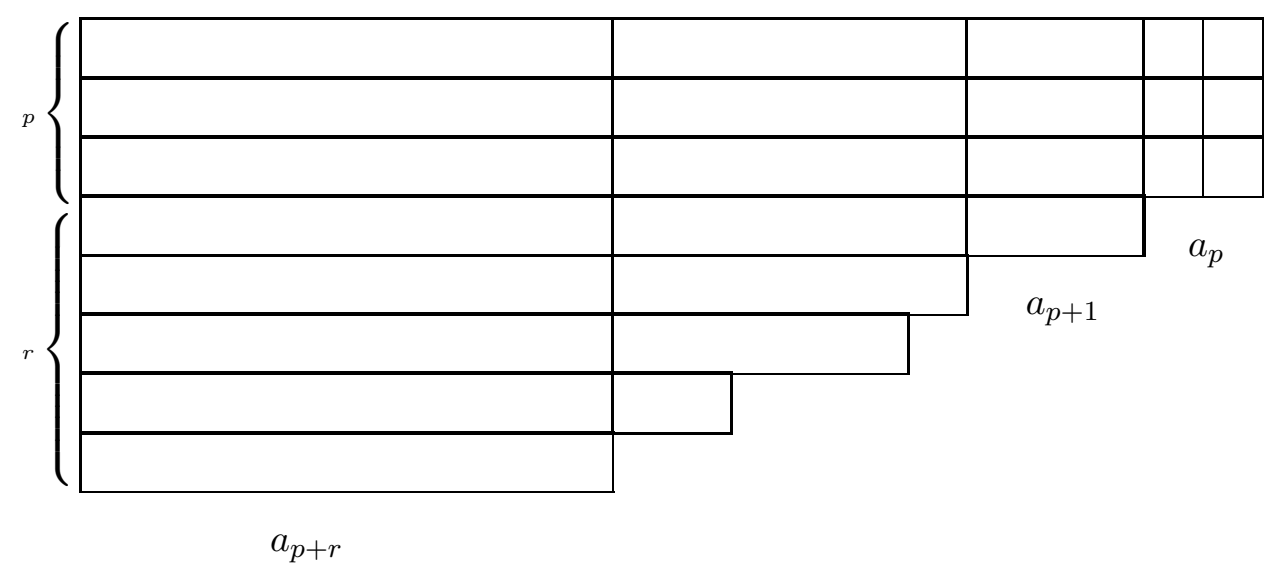

From a purely algebraic point of view representations of superconformal groups in four dimensions are determined by the following set of labels: $\left(J_{1}, J_{2}, L, R ; a_{1}, a_{2}, \ldots a_{N-1}\right)$, where $J_{1}, J_{2}$ are spins, $L, R$ are charges corresponding to dilations and R-symmetry respectively while the $a^{\prime} s$ are $\mathfrak{s l}(N)$ Dynkin labels (see, e.g., [5, 17] for reviews). For representations of the above type $J_{1}=J_{2}=0$, as are the Dynkin labels $a_{1}, \ldots a_{p-1} ; a_{N-q+1} \ldots a_{N-1}$, while the $L$ and $R$ charges are, except when either $p$ or $q=0, N \neq 4$ determined by these labels. The representations that occur belong to series $\mathrm{C}$ with $J_{1}=J_{2}=0$ for which one has

$$
L=m_{1} ; \quad R=\frac{2 m}{N}-m_{1}
$$

where 


$$
m:=\sum_{k=1}^{N-1} k a_{k} ; \quad m_{1}:=\sum_{k=1}^{N-1} a_{k}
$$

Note that $m$ is the total number of boxes in the $\mathfrak{s l}(N)$ Young tableau, while $m_{1}$ is the number of boxes in the first row. When $q=0, N \neq 4$, one has the weaker condition

$$
L-R=2 m_{1}-\frac{2 m}{N}
$$

while if $p=0, N \neq 4$, one finds

$$
L+R=\frac{2 m}{N}
$$

Both of these conditions are compatible with either series $\mathrm{C}$ or series $\mathrm{B}$. In the latter case one has, for zero spins, $L \geq m_{1}+1$, together with either (34) or (35). In these cases one more label ( $L$ or $R$ ) has to be specified in order to fix the representation. For $N=4$ the same formulae hold, but now $R=0$ as well. In particular, this means that the representations are fully determined by the Dynkin labels even if $p$ or $q=0$. It should be noted that there is a second possibility for $N=4$ when the group is $S L(4 \mid 4)$ and not $P S L(4 \mid 4)$; this case is the same as general $N$.

To demonstrate the above explicitly, we first note that, in the basis in which we are working, the matrices representing $L$ and $R$ are

$$
L=\frac{1}{2}\left(\begin{array}{l|l|l}
-1_{2} & & \\
\hline & & \\
& & 1_{2}
\end{array}\right)
$$

and

$$
R=\frac{1}{2}\left(\begin{array}{l|l|l}
1_{2} & & \\
\hline & \frac{4}{N} 1_{N} & \\
& & 1_{2}
\end{array}\right)
$$

As we have mentioned, for most of these representations, fixing $(p, q)$ and the Dynkin labels determines the values of $L$ and $R$ as well. The key point is that, as we remarked above, factors such as $\mathfrak{s l}(2 \mid p)$ must be represented trivially in order for the fields we are interested in to have $J_{1}=J_{2}=0$. To put it another way, the sub-algebra $\mathfrak{g l}(2 \mid p)$ is represented by its supertrace. This means that any element with vanishing supertrace becomes zero when acting on fields of interest. In particular, 


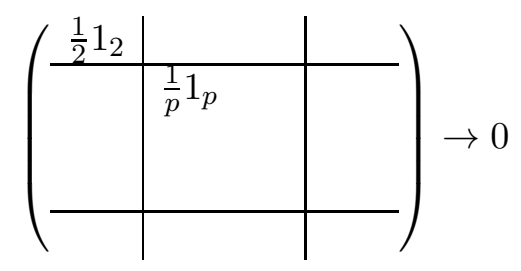

and, similarly

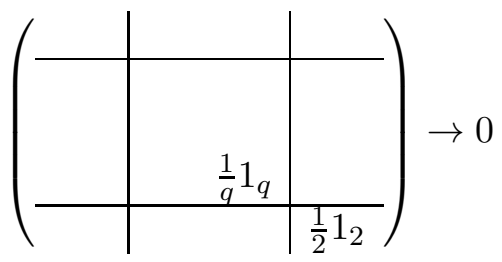

The operator $1_{p}$ on the representation in question counts the total number of boxes in the first $p$ rows, of the Young tableau (31), while the operator $1_{N}$ counts the total number of boxes in the diagram. If we denote the field by $A$, then $1_{p} A=p m_{1} A, 1_{N} A=m A$; since the tableau (31) has boxes only in the first $N-q$ rows it follows that $1_{q} A=0$.

It might be thought that when either $p$ or $q$ equals 2 there could be an extra charge due to the fact that $\mathfrak{s l}(n \mid n)$ is not simple since the unit matrix has vanishing supertrace. However, for vector representation spaces which do not carry any indices for this supergroup, one can show that the unit matrix must be represented by zero (because the anticommutator of two odd generators includes it) and this means that the above rules for evaluating $L$ and $R$ apply in this case, too.

In $N=4$ the superconformal group can be taken to be either $S L(4 \mid 4)$ or $P S L(4 \mid 4)$. The latter group is simple and is the one which is relevant for SYM, while the former is needed for certain representations such as the spin 3/2 supermultiplet. In the second case case it is still easier to work with $S L(4 \mid 4)$ and impose the constraint $R=0$ to get to $P S L(4 \mid 4)$. Note that in either case R-symmetry does not act on the coordinates.

We shall now go through the various cases that can arise, explicitly evaluating $L$ and $R$ for the representations in question:

\section{The generic case: $p, q, r \neq 0$}

In this case, the residual symmetry algebra acting on the representation space of the isotropy group $V$ is $\mathfrak{s l}(r) \oplus \mathbb{C}^{2}$. Using the above rules for evaluating the unit matrices $1_{N}, 1_{p}, 1_{q}$, we find, as expected,

$$
L=m_{1} ; \quad R=\frac{2 m}{N}-m_{1}
$$

The representation belongs to series $\mathrm{C}$.

\section{2. $p, q \neq 0 ; r=0$}

In this case the residual symmetry algebra is simply $\mathbb{C}$ and the Dynkin diagram reduces to 


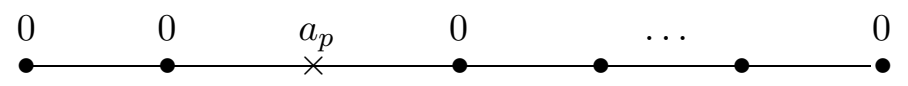

so that all the labels are zero except for $a_{p}$. $L$ and $R$ are given by the same formulae with $m=p a_{p} ; m_{1}=a_{p}$. The Young tableau is

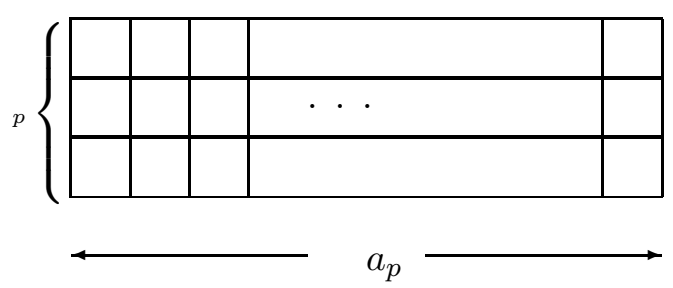

3. $p \neq 0, q=0$

The Dynkin diagram now has the form:

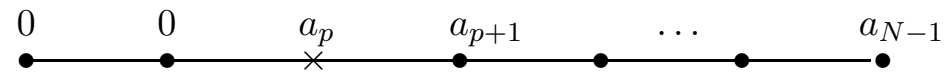

In this case the Levi subalgebra is $s(\mathfrak{g l}(2 \mid p) \oplus \mathfrak{g l}(N-p) \oplus \mathfrak{g l}(2))$, where the last term arises from the fact that $q=0$. In this case the constraint (39) is lost, and one therefore only finds that

$$
L-R=2 m_{1}-\frac{2 m}{N}
$$

This is compatible with both series B and C representations.

\section{4. $p=0, q \neq 0$}

The Dynkin diagram now has the form:

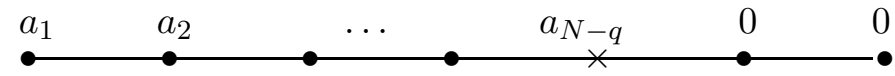

In this case the Levi subalgebra is $s(\mathfrak{g l}(2 \mid q) \oplus \mathfrak{g l}(N-q) \oplus \mathfrak{g l}(2))$, where the last term now arises because $p=0$. Again the constraint (38) is lost, and one therefore only finds that

$$
L+R=\frac{2 m}{N}
$$

This is also compatible with series B and C.

For both $(p, q)=(p, 0)$ and $(p, q)=(0, q)$ there is the possibility of having superfields with all Dynkin labels equal to zero. These fields just behave as holomorphic functions with regard 
to the internal space and therefore do not depend on the coordinates of the internal space by compactness. G-analyticity then implies that they are chiral or anti-chiral scalar fields (for $(0, q)$ or $(p, 0)$ respectively). For $L=1$ such superfields are not irreducible and satisfy an additional constraint, as we shall discuss in the next section, but for other (higher) values of $L$ they are irreducible superconformal fields with $L= \pm R$.

The remaining examples all refer to $N=4$ with the group $P S L(4 \mid 4)$.

\section{5. $(p, q)=(1,3)$}

This is a particular example of $(N, p, N-p)$ analytic superspace. In principle, one would expect to have Dynkin diagrams of the form

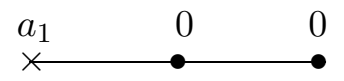

but the imposition of $R=0$ implies that $a_{1}=0$, so that there are no short representations on this space.

\section{6. $(p, q)=(2,2)$}

The Dynkin diagram is:

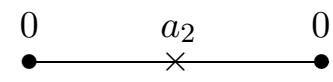

and this is compatible with $R=0$.

\section{7. $(p, q)=(1,2)$}

We expect to have

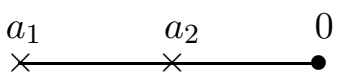

but $R=0$ implies that $a_{1}=0$; hence there are no new $(1,2)$ representations, since the ones that are allowed can be formulated on $(2,2)$ superspace.

\section{8. $(p, q)=(1,1)$}

In this case we could expect to have arbitrary representations of $\mathfrak{s l}(4)$,

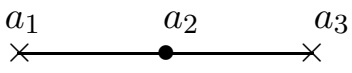


However, $R=0$ implies that $a_{1}=a_{3}$.

\section{9. $(p, q)=(p, 0)$}

The possible diagrams are

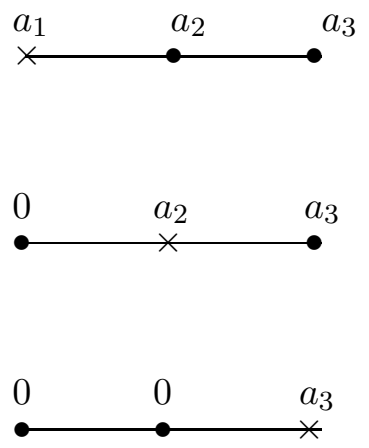

for $p=1,2,3$ respectively. In all cases we find that

$$
L=2 m_{1}-\frac{m}{2}
$$

because $R=0$ and $q=0$. This can be rewritten as

$$
L=m_{1}+\frac{1}{2}\left(a_{1}-a_{3}\right)
$$

Since the superfields under consideration are bosonic it follows that $L$ must be integral, and hence $a_{1}-a_{3}=2 b$, so that

$$
L=m_{1}+b \geq m_{1}+1 \text { for } b \geq 1
$$

There are therefore no $p=3$ representations, while for $p=2$ only $a_{3}=0$ is allowed. Finally, for $p=1$ we have $a_{1}-a_{3}=2 b$ so that, for $b \neq 0$ we have series B with $L=m_{1}+b$, while if $b=0$ we have series $\mathrm{C}$ with $a_{1}=a_{3}$.

10. $(p, q)=(0, q)$

The diagrams are the same as in the previous case, but now they correspond to $q=3,2,1$ respectively. In all cases we have

$$
L=\frac{m}{2}=m_{1}+\frac{1}{2}\left(a_{3}-a_{1}\right)
$$

There are no representations for $q=3$, for $q=2$ we can only have $a_{2} \neq 0$ and for $q=1$ we can have series B with $L=m_{1}+b$ and $a_{3}-a_{1}=2 b \geq 2$, or series $\mathrm{C}$ with $L=m_{1}, a_{1}=a_{3}$. 
We conclude this survey of the various short representations with a brief discussion of the general case. For the generic case, we can have representations of the following type:

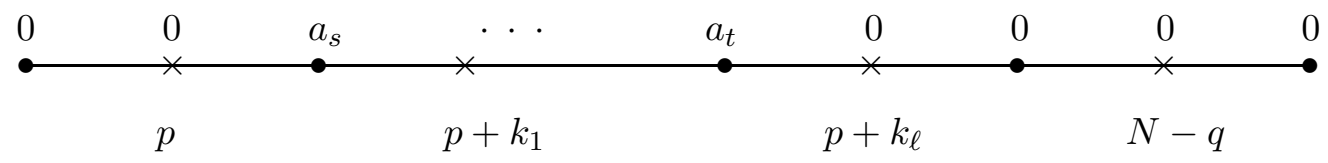

This diagram is read as follows: the crosses determine the internal parabolic subalgebra of $\mathfrak{s l}(N)$, the corresponding internal flag manifold being $\mathbb{F}_{p, p+k_{1}, \ldots p+k_{\ell}, N-q}(N)$; the two outer crosses, at the $p$ th and $(N-q)$ th nodes, determine the explicitly realised G-analyticity to be of type $(p, q)$, and the non-zero Dynkin labels are $a_{s} \ldots a_{t}$ where $s \geq p$ and $t \leq N-q$. If $s=p, t=N-q$ the representation is maximally efficient, but otherwise this is not the case. This holds if neither $p$ nor $q$ is zero. The representation of the superconformal group is fixed by the diagram since $L$ and $R$ can be determined and the spins are zero. If either of $p$ or $q$, say $q$, equals zero, then the only restriction is that the left-most cross must be placed at node $p$, with $s \geq p$. As we have seen, in this case the representation is only fully determined when an extra label is specified unless $N=4$ and the group is $P S L(4 \mid 4)$ where the fact that $R=0$ implies that no extra label is required.

Finally, we note that any short representation can be realised on $(1,0)$ superspace, while any representation from series $C$ can be realised on $(1,1)$ superspace, although these realisations will not be maximally efficient in general.

\section{Massless supermultiplets}

On-shell massless supermultiplets with maximal helicity $s$, where $\left[\frac{N}{2}\right] \leq 2 s<N,\left(\left[\frac{N}{2}\right]\right.$ denotes the nearest integer greater than or equal to $\frac{N}{2}$ ), are described in (real) super Minkowski space, $M$, by superfields $W$ which have $p=2 s$ totally antisymmetric internal indices and which satisfy [1], 8]

$$
\begin{aligned}
\bar{D}_{\dot{\alpha}}^{i} W_{j_{1} \ldots j_{p}} & =\frac{p(-1)^{p-1}}{N-p+1} \delta_{\left[j_{1}\right.}^{i} \bar{D}_{\dot{\alpha}}^{k} W_{\left.j_{2} \ldots j_{p}\right] k} \\
D_{\alpha i} W_{j_{1} \ldots j_{p}} & =D_{\alpha[i} W_{\left.j_{1} \ldots j_{p}\right]}
\end{aligned}
$$

For each such superfield there is a conjugate superfield $\tilde{W}_{i_{1} \ldots i_{N-p}}$ defined by

$$
\tilde{W}_{i_{1} \ldots i_{N-p}}=\frac{1}{p !} \varepsilon_{i_{1} \ldots i_{N-p} j_{N-p+1} \ldots j_{N}} \bar{W}^{j_{N-p+1} \ldots j_{N}}
$$

The conjugate superfield obeys similar constraints:

$$
\bar{D}_{\dot{\alpha}}^{i} \tilde{W}_{j_{1} \ldots j_{N-p}}=\frac{(N-p)(-1)^{N-p-1}}{p+1} \delta_{\left[j_{1}\right.}^{i} \bar{D}_{\dot{\alpha}}^{k} \tilde{W}_{\left.j_{2} \ldots j_{N-p}\right] k}
$$




$$
D_{\alpha i} \tilde{W}_{j_{1} \ldots j_{N-p}}=D_{\alpha[i} \tilde{W}_{\left.j_{1} \ldots j_{N-p}\right]}
$$

When $s=\frac{1}{4} N$, the multiplet is self-conjugate:

$$
\bar{W}^{i_{1} \ldots i_{p}}=\frac{1}{p !} \varepsilon^{i_{1} \ldots i_{p} j_{1} \ldots j_{p}} W_{j_{1} \ldots j_{p}}
$$

For each $N$ there is therefore a range of scalar superfields $W_{i_{1} \ldots i_{p}}$ antisymmetric on $p$ internal indices with $p$ ranging from 1 to $N-1$ all of which obey the constraints (57). We can extend this to $N$, since for such a superfield the constraints (57) imply that it is anti-chiral. Its conjugate has no indices and is chiral. Such a chiral field describes an on-shell massless supermultiplet (with maximum spin $N / 2$ ) if it satisfies the additional constraint 18]

$$
D_{\alpha i} D_{j}^{\alpha} W=0
$$

Note that this equation is only superconformal if the dilation weight $L=1$; for other values of $L$ a chiral field is superconformally irreducible and (61) should not be imposed.

The superfields $W_{i_{i} \ldots i_{p}}$ can be naturally described as CR-analytic fields on $(N, p, N-p)$ harmonic superspaces [9]. On $M \times S U(N)$ define the superfield $W^{(p)}$ by

$$
W^{(p)}=\frac{1}{p !} \varepsilon^{r_{1} \ldots r_{p}} u_{r_{1}}{ }^{i_{1}} \ldots u_{r_{p}}{ }^{i_{p}} W_{i_{1} \ldots i_{p}}
$$

then equation (57) implies that $W^{(p)}$ satisfies the G-analyticity constraints

$$
\begin{aligned}
D_{\alpha r} W^{(p)} & =0 \\
\bar{D}_{\dot{\alpha}}^{r^{\prime}} W^{(p)} & =0
\end{aligned}
$$

and the H-analyticity constraint,

$$
D_{r}{ }^{\prime} W^{(p)}=0
$$

In addition $W^{(p)}$ is clearly equivariant with respect to the isotropy group $S(U(p) \times U(N-p))$ of the internal flag manifold $\mathbb{F}_{p, N-p}(N)$, which in this case is the Grassmannian of $p$-planes in $\mathbb{C}^{N}$, $\mathbb{G}_{p}(N)$. Furthermore, an equivariant field with the same charge as $W^{(p)}$ satisfying the above analyticity constraints is equivalent to a superfield on super Minkowski superspace satisfying (57). We can apply this construction to the entire family of scalar superfields with $p$ varying from 0 to $N$, bearing in mind that the chiral fields satisfy the additional constraint (61).

Under harmonic conjugation the superfield $W^{(p)} \mapsto \widetilde{\left(W^{(p)}\right)}=W^{(N-p)}$.

In complexified superspace, there are corresponding fields $W^{(p)}$ and $\tilde{W}^{(p)} \operatorname{defined}$ on $(N, p, N-p)$ and $(N, N-p, p)$ analytic superspace respectively. Furthermore, it is possible to extend harmonic conjugation to this case straightforwardly. The Dynkin diagram for $W^{(p)}$ is 


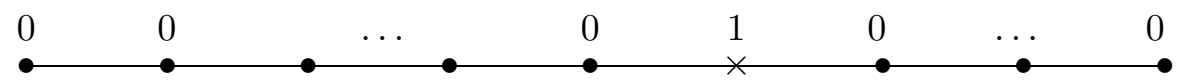

where the single non-zero label is above the $p$ th node, and thus corresponds to the representation of $\mathfrak{s l}(N)$ on $\wedge^{P}\left(\mathbb{C}^{N}\right)$. The diagram for $\tilde{W}^{(p)}$ is similar, but with $p$ replaced by $N-p$.

Although $W^{(p)}$ provides the most efficient realisation of this representation, the maximum spin for $W^{(p)}$, when $p \geq N / 2$, is in fact only $\left(0, \frac{p}{2}\right)$, and not $\left(\frac{N-p}{2}, \frac{p}{2}\right)$; these representations are therefore ultra-short. For $\tilde{W}^{(p)}$ the maximum spin is $\left(\frac{p}{2}, 0\right)$. In the self-conjugate case, $W^{(p)}$ contains both $\left(0, \frac{p}{2}\right)$ and $\left(\frac{p}{2}, 0\right)$.

There are many other ways of representing such multiplets which are "less efficient" in that the superspaces have more odd coordinates. Following the discussion at the end of the previous section we can simply place crosses where we like on the above Dynkin diagram with the restrictions that the cross furthest to the left must have node number $k$ less or equal to $p$ and the cross furthest to the right must have node number $l$ at least equal to $p$. The corresponding superspace will then have G-analyticity of type $(k, l)$. For example, any such field (excluding $p=0, N)$ can be realised with $(1,1)$ G-analyticity on an internal flag defined by the Borel subgroup (i.e. crosses at all nodes).

To illustrate this procedure we consider the $N=4$ Maxwell supermultiplet which is represented on $N=4$ super Minkowski space by the familiar self-conjugate Sohnius superfield $W_{i j}$ [32]. The possible realisations of this superfield have G-analyticities $(2,2),(1,1)$ and $(1,0)$. The $(2,2)$ superfield is the one introduced in [9]. The $(1,1)$ superfield can be written $W_{1 r}, r \in\{2,3\}$; in $(4,1,1)$ harmonic superspace it obeys the constraints

$$
D_{\alpha 1} W_{1 r}=\bar{D}_{\dot{\alpha}}^{4} W_{1 r}=0
$$

Under harmonic conjugation we have

$$
W_{1 r} \mapsto \tilde{W}^{4 r}=\epsilon^{r s} W_{1 s}
$$

The $(1,0)$ version is a superfield $W_{1 r}, r \in\{2,3,4\}$ satisfying

$$
D_{\alpha 1} W_{1 r}=0
$$

while the $(0,1)$ version is a superfield $W_{R S}, R \in 1,2,3$ obeying

$$
\bar{D}_{\dot{\alpha}}^{4} W_{R S}=0
$$

Under harmonic conjugation we find

$$
W_{1 r} \mapsto \tilde{W}^{4 R}=\frac{1}{2} \epsilon^{R S T} W_{S T}
$$


Due to self-conjugacy, the constraint (69) together with harmonic analyticity is sufficient to reproduce the standard constraints obeyed by the Sohnius superfield.

The above superfields have been defined on $(4,1,1)$ and $(4,1,0)$ or $(4,0,1)$ superspaces which have the smallest possible internal flags. It is possible to relax this; for example we could use the maximal flag space determined by the Borel subalgebra. In the $(1,1)$ case we would then split the indices as $I=1,2,3,4$; this enables us to define the field $W_{12}$ which is $(1,1)$ G-analytic and $\mathrm{H}$-analytic on $\mathbb{F}_{B}$. Note that this field satisfies further first-order differential constraints; it is actually $(2,2)$ G-analytic and is also annihilated by $D_{2}{ }^{1}$ and $D_{4}{ }^{3}$

\section{$5 \quad$ Tensor products}

General short multiplets can be obtained as tensor products of the massless supermultiplets in a straightforward fashion. If one wishes to find such a short multiplet with $(p, q)$ analyticity the optimal way to construct it from massless multiplets is to use realisations of the latter with the same analyticity. One then multiplies an appropriate number of massless multiplets together to get the desired representation. Because the massless multiplets will in general transform under non-trivial representations of the internal symmetry algebra $(\mathfrak{s l}(r)$ in the case of $(N, p, q)$ superspace), it will be necessary to project out an irreducible representation of this algebra in order to obtain the desired irreducible short representation.

\subsection{Generating all short representations}

There is a very simple way of generating all possible short representations from the set of available massless supermultiplets for a given $N$. Consider first those belonging to series $\mathrm{C}$. We realise each of the superfields $W^{(p)}$ on $(N, 1,1)$ superspace with $\mathbb{F}_{B}$ as the internal flag space. Thus we have superfields $W_{1}, W_{12}, W_{123}, \ldots W_{123 \ldots N-1}$ all of which are G-analytic with respect to $D_{\alpha 1}$ and $\bar{D}_{\dot{\alpha}}^{N}$. Let $\left(a_{1}, \ldots a_{N-1}\right)$ be the Dynkin labels of the representation we are interested in and simply form the product

$$
A=\prod_{k=1}^{N-1}\left(W_{12 \ldots k}\right)^{a_{k}}
$$

The leading component of this superfield transforms according to the irreducible representation of $S L(N)$ specified by the Dynkin labels. The dilation weight is just equal to the sum of the Dynkin labels, that is $m_{1}$, since each underlying field has weight 1.

This construction of a general representation also illustrates what happens when the Dynkin labels are restricted. For example, suppose that the first $p-1$ and the last $q-1$ labels are zero, then the only superfields present in the above product will be $W_{12 \ldots p}, \ldots W_{12 \ldots N-q}$. Each

of these superfields is G-analytic with respect to $D_{\alpha 1} \ldots D_{\alpha p}$ and $\bar{D}_{\dot{\alpha}}^{N-q+1} \ldots \bar{D}_{\dot{\alpha}}^{N}$; furthermore, as well as being $\mathrm{H}$-analytic on $\mathbb{F}_{B}$ it satisfies further harmonic anti-analyticity conditions and can therefore be thought of as a superfield on $\mathbb{F}_{p, p+1, p+2, \ldots N-q-1, N-q}(N)$ superspace. 
For series $\mathrm{B}$ representations we can work on $(N, 0,1)$ superspace again with $\mathbb{F}_{B}$ as the internal flag manifold. We use the same family of superfields as before augmented with $W_{o}$ which satisfies $\bar{D}_{\dot{\alpha}}^{N} W_{o}=0$ together with the additional condition (61). We can then form the product superfield

$$
A^{\prime}=\left(W_{o}\right)^{b} \prod_{k=1}^{N-1}\left(W_{12 \ldots k}\right)^{a_{k}}
$$

where $b$ is a positive integer. The $L$ weight of this field is $b$ plus the dilation weight of the previous example, so $L=m_{1}+b$. Likewise the R-symmetry weight differs from the previous example by $-b$, so $R=2 m / N-\left(m_{1}+b\right)$ and therefore $L+R=2 m / N$ as required. In general this superfield will only be annihilated by $\bar{D}_{\dot{\alpha}}^{N}$, but if the last $q-1$ Dynkin labels vanish, the superfield will be defined on $(N, 0, q)$ superspace.

If the field $A$ of (72) is multiplied by products of the anti-chiral field with $L=1, R=1$ we get the $\mathrm{B}$ series of representations on $(N, 1,0)$ superspace with $L-R=2 m_{1}-2 m / N$.

\subsection{Products of a given massless multiplet}

In order to find the short representation composites that can be constructed using a given underlying massless supermultiplet one first of all computes the different Young tableau that can arise by multiplying together the tableaux for $k$ factors of $W$ and $l$ factors of $\tilde{W}$. For series $\mathrm{C}$, these tableaux need to be subjected to the following restrictions: firstly, there should be $k+l$ boxes in the first row, secondly, there should be a total of $p k+(N-p) l$ boxes with at most $N-1$ in the first column and thirdly, a number of representaions will be absent due to symmetrisation. In the case of series $\mathrm{B}$, one can either have columns with $N$ boxes, in which case after these have been discarded the remaining tableaux will clearly have fewer than $p k+(N-p) l$ boxes, or one is still restricted to at most $N-1$ boxes in a column, but with the constraint that there should be $k+l$ boxes in the first row dropped. The problem of restrictions due to symmetrisation can be overcome if one is allowed to use different copies of the same underlying multiplet.

As a simple example consider the $N=3$ Maxwell multiplet which is described by an on-shell superfield $W_{i j}$ of the above type. The corresponding $W$ is CR-analytic on $(3,2,1)$ harmonic

superspace, and its conjugate $\tilde{W}$ is CR-analytic on $(3,1,2)$ harmonic superspace. Both of them can be realised as CR-analytic superfields on $(3,1,1)$ superspace, with

$$
W_{12}=u_{1}{ }^{i} u_{2}^{j} W_{i j} ; \quad \tilde{W}_{1}=u_{1}{ }^{i} \tilde{W}_{i}
$$

On $(3,2,1)$ superspace, the products of $W$ with itself have maximum spins $\frac{1}{2}, 1$, and corresponding tableaux with 2 rows of the same length. The simplest of these supermultiplets, $S$, say, has its leading component in the 6-dimensional representation of $S U(3)$. In order to construct the energy-momentum tensor multiplet one has to take the product of $W$ and $\tilde{W}$ on $(3,1,1)$ superspace. This multiplet, $T$, say, has maximum spin $(1,1)$ and its leading component is in the 8-dimensional representation of $S U(3)$. The multiplets $T$ and $S$ are the $N=3$ "components" of the $N=4$ supercurrent. (See [17] for more details of $N=3$ mulitplets.) 
An interesting set of multiplets can be found for supergravity theories with $N \geq 5$. The basic scalar superfield with maximum spin 2 has four indices and is self-conjugate in $N=8$. The analogue of the energy-momentum tensor in some senses for this multiplet is the Bel-Robinson

tensor $\left(C_{\alpha \beta \gamma \delta} \bar{C}_{\dot{\alpha} \dot{\beta} \dot{\gamma} \dot{\delta}}+\ldots\right.$ in two-component spinor notation, where $C$ is the Weyl spinor $)$. This spin 4 field is the highest component of the composite multiplet obtained by multiplying $W$ and its conjugate. This superfield lives naturally on $(N, N-4, N-4)$ superspace. If we square it, we obtain the three-loop supergravity counterterm supermultiplet [33]. In $N=8$ this is simply $W^{4}$ since $W$ is self-conjugate [9].

\section{$5.3 \mathrm{~N}=4$ composites}

We conclude this discussion of composite operators with some comments on $N=4 \mathrm{SYM}$ since this is of most interest in the AdS/CFT context. The superconformal group in this case is $P S U(2,2 \mid 4)$ and these fields were discussed from a different perspective in 17].

We begin with Maxwell multiplets which we denote by $A, B, C$, etc. If we regard these as superfields on $(4,2,2)$ superspace, the only composites we can build are of the form $A^{k}$ with Dynkin labels $(0, k, 0)$ and highest spin $(1,1)$. On $(1,1)$ superspace we can generate all the series $\mathrm{C}$ representations using two fields $A_{1 r}, B_{1 r}$, where $r \in\{2,3\}$. The product $A^{k} B^{l}$, where we may assume $k \geq l$, gives rise to a series of representations with Dynkin labels $(n, k+l-2 n, n)$ where $n=0,1, \ldots l$. The point here is that products of $A$ with itself must be symmetric and can therefore have tableaux with only two rows. To get three rows it is necessary to introduce a second field $B$. Finally, to get representations from series B one has to use a third field $C$ and work on $(1,0)$ superspace. In this manner one can generate tableaux with four rows, although one then discards the columns which do have four rows.

In the non-Abelian theory the field strength superfield $W$ takes its values in the Lie algebra of the gauge group which we shall take to be $S U\left(N_{c}\right)$; it is not itself a CR-analytic superfield on $(4,2,2)$ harmonic superspace, rather it is H-analytic and covariantly G-analytic [9]. However, gauge-invariant products of powers of $W$ are CR-analytic and hence can be used to construct short representations of the $N=4$ superconformal group. The simplest series is the KK series $A_{k}:=\operatorname{Tr}\left(W^{k}\right)$; this series of operators on $(4,2,2)$ space has maximum spin $(1,1)$ and includes the supercurrent for $k=2$ [9, 10]. To obtain other short representations from series $\mathrm{C}$ we consider the field strength tensor as a $(4,1,1)$ superfield $W_{1 r}, r \in\{2,3\}$. We can then form the double trace operators $\operatorname{Tr}\left(W_{1 r}^{k}\right) \operatorname{Tr}\left(W_{1 s}^{l}\right)$, which of course decompose into irreducible representations under $\mathfrak{s l}(2)$. Finally, we can obtain series B representations by taking triple traces of a similar type using the basic superfield $W_{1 r}, r \in\{2,3,4\}$ which is covariantly G-analytic on $(4,1,0)$ superspace. Each factor in these multi-trace operators, if we assume the gauge-group trace to be symmetrised, is one of the operators in the $A_{k}$ series, so that another way of obtaining these operators is as products of these. For series $\mathrm{C}$ has to write these operators in $(4,1,1)$ superspace, thus $A_{k}=\operatorname{Tr}\left(W^{k}\right)$ is replaced by $A_{r_{1} \ldots r_{k}}:=\operatorname{Tr}\left(W_{1 r_{1}} \ldots W_{1 r_{k}}\right)$. One can then form the products $A_{r_{1} \ldots r_{k}} A_{s_{1} \ldots s_{l}}$ which can subsequently be decomposed into irreducible representations under $S U(2)$. Similarly the triple trace operators can be viewed as products of sets of three $A_{k}$ operators written as superfields on $(4,1,0)$ superspace. The multi-trace operators are also discussed in [17. 
It would seem that further gauge-invariant operators could be constructed using the structure constants, ; for example, on $(1,0)$ superspace, there is an operator $\varepsilon^{r s t} \operatorname{tr}\left(W_{1 r} W_{1 s} W_{1 t}\right)$ where the trace is no longer symmetrised.

\section{Superconformal transformations on harmonic superspace}

The short representations obtained by parabolic induction in complex superspaces transform under the superconformal group in the standard way. These transformations can then be adapted to real spacetime. However, if one is interested in real harmonic superspaces in the GIKOS formalism it is perhaps just as easy to derive the explicit transformation rules by other means. This is what we shall do in this section. Most of the results given here were obtained previously in [6], but the derivation is perhaps slightly simpler. We start from superconformal transformations on super Minkowski space, extend these in a simple way to $M \times S U(N)$ and then make a final modification in order to preserve analyticity.

We recall that a superconformal transformation on super Minkowski space is a diffeomorphism which preserves the odd tangent space (spanned by the odd covariant derivatives). Dually, such a transformation can be thought of as one which preserves the even cotangent bundle spanned

by the differential forms $E^{\alpha \dot{\alpha}}:=d x^{\alpha \dot{\alpha}}+\frac{i}{2} d \theta^{\alpha i} \bar{\theta}_{i}^{\dot{\alpha}}+\frac{i}{2} d \bar{\theta}_{i}^{\dot{\alpha}} \theta^{\alpha i}$. An infinitesimal such transformation is generated by a superconformal Killing vector $V$ where

$$
V=F^{\alpha \dot{\alpha}} \partial_{\alpha \dot{\alpha}}+f^{\alpha i} D_{\alpha i}-\bar{f}_{i}^{\dot{\alpha}} \bar{D}_{\dot{\alpha}}^{i}
$$

with

$$
D_{\alpha i} F_{\beta \dot{\beta}}=-i \varepsilon_{\alpha \beta} \bar{f}_{\dot{\beta} i}
$$

so that

$$
f^{\alpha i}=\frac{i}{2} \bar{D}_{\dot{\alpha}}^{i} F^{\alpha \dot{\alpha}}
$$

Thus such a vector field is determined by the function $F^{\alpha \dot{\alpha}}$ obeying the above constraint. The Lie algebra can therefore be presented in terms of $F$,

$$
\left[V_{F}, V_{F^{\prime}}\right]=V_{\left[F, F^{\prime}\right]}
$$

where

$$
\left[F, F^{\prime}\right]^{\alpha \dot{\alpha}}:=F^{\prime \prime \alpha \dot{\alpha}}=\left(F \cdot \partial F^{\prime \alpha \dot{\alpha}}-i f^{\alpha i} \bar{f}_{i}^{\prime} \dot{\alpha}\right)-\left(F \leftrightarrow F^{\prime}\right)
$$

It is not difficult to verify that the superfield $F$ subject to (76) does indeed specify a superconformal transformation. In $N=4$ there is an additional constraint, namely 


$$
D \cdot f+\bar{D} \cdot \bar{f} ;=D_{\alpha i} f^{\alpha i}+\bar{D}_{\dot{\alpha}}^{i} \bar{f}_{i}^{\dot{\alpha}}=0
$$

For general $N$ one has the identity

$$
\partial \cdot F-\frac{2}{N}(D \cdot f-\bar{D} \cdot \bar{f})=0
$$

and we identify the dilation and R-symmetry parameters $L$ and $R$ by

$$
\begin{aligned}
\partial \cdot F & =4 L+\ldots \\
D \cdot f+\bar{D} \cdot \bar{f} & =-2 i(N-4) R, \mathrm{~N} \neq 4
\end{aligned}
$$

The $N=4$ constraint ( 80 ) has the geometrical consequence that the $N=4$ chiral measure is superconformally invariant, since it implies that $\partial \cdot F-D \cdot f=0$.

The above transformation can be extended to a transformation of $M \times S U(N)$. The corresponding Killing vector in this case has the form

$$
\mathcal{V}=V_{o}+V_{u}
$$

where $V_{o}$ is a superconformal Killing vector on super Minkowski space and

$$
V_{u}:=f_{I}^{J} D_{J}^{I}
$$

with

$$
f_{I}^{J}=\frac{1}{2}\left(D_{\alpha I} f^{\alpha J}-\frac{1}{N} \delta_{I}^{J} D_{\alpha K} f^{\alpha K}\right)
$$

The parameter $f_{I}{ }^{J}$ is the parameter superfield $f_{i}{ }^{j}$ with indices converted to capital ones by means of $u$ 's. The leading component of $f_{i}{ }^{j}$ is the $\mathfrak{s u}(N)$ parameter.

It is straightforward to verify that $\mathcal{V}$ gives a representation of the superconformal algebra.

If $A$ is an equivariant superfield on $M \times S U(N)$ then it would be natural to look for a transformation rule of the form $\delta A=\mathcal{V} A$. This preserves $\mathrm{H}$-analyticy, but not preserve G-analyticity. This deficiency can be overcome by including an extra term in the transformation rule. This term is constructed using the chiral function $K$ defined by

$$
K=\frac{1}{4} \partial \cdot F+\frac{1}{2(N-4)}(D \cdot f+\bar{D} \cdot \bar{f})
$$

which is normalised so that its leading component is $S-i R$. It is also proportional to the transformation factor of the chiral measure. A term of the form $(a K+b \bar{K}) A$ can be added to the transformation rule for $A$ because 


$$
V_{F} K_{F^{\prime}}-V_{F^{\prime}} K_{F}=K_{\left[F, F^{\prime}\right]}
$$

Moreover, it is the only available function which preserves H-analyticity.

We consider a field $A$ which has $(p, q) \mathrm{G}$-analyticity and which is H-analytic in the sense of $(N, p, q)$ harmonic superspace. As before, we set $I=\left(r, r^{\prime \prime}, r^{\prime}\right)$. The field $A$ satisfies the Ganalyticity conditions

$$
D_{\alpha r} A=\bar{D}_{\dot{\alpha}}^{r^{\prime}} A=0
$$

as well as the H-analyticity conditions

$$
D_{r}{ }^{s^{\prime}} A=D_{r}{ }^{s^{\prime \prime}} A=D_{r^{\prime \prime}} s^{\prime} A=0
$$

$A$ cannot transform under $S U(p)$ (acting on $r$ ) or $S U(q)$ (acting on $r^{\prime}$ ) because this is incompatible with G-analyticity. Thus we find

$$
\begin{aligned}
V_{u} A= & f_{r}^{s^{\prime}} D_{s^{\prime}}{ }^{r} A+f_{r} s^{\prime \prime} D_{s^{\prime \prime}}{ }^{r} A+f_{r^{\prime \prime}} s^{\prime} D_{s^{\prime}} r^{\prime \prime} A+f_{r^{\prime \prime}} s^{\prime \prime} \hat{D}_{s^{\prime \prime}} r^{\prime \prime} A \\
& +f_{o} D_{o} A+f_{o}^{\prime} D_{o}^{\prime} A+f_{o}^{\prime \prime} D_{o}^{\prime \prime} A
\end{aligned}
$$

The first three terms involve the coset derivatives of the internal flag manifold and the absence of their conjugates reflects the H-analyticity of the field $A$. The fourth term takes care of the transformation properties of $A$ under $S U(r)\left(\hat{D}_{r^{\prime \prime}} r^{\prime \prime}=0\right)$. The next two terms are left over from $f_{r}{ }^{s} D_{s}{ }^{r} A$ and $+f_{r^{\prime}} s^{\prime} D_{s^{\prime}} r^{\prime} A$ which can only be non-trivial for the trace parts, and the final term arises from removing the trace from $D_{r^{\prime \prime}} s^{\prime \prime}$. In the above we have used the definition

$$
D_{o}=\frac{1}{p} D_{r}^{r} ; \quad f_{o}=f_{r}^{r}
$$

with similar definitions for primed and double-primed indices. The fact that we are working with $S U(N)$ then implies

$$
\begin{array}{r}
p D_{o}+q D_{o}^{\prime}+r D_{o}^{\prime \prime}=0 \\
f_{o}+f_{o}^{\prime}+f_{o}^{\prime \prime}=0
\end{array}
$$

A short calculation shows that

$$
\begin{aligned}
D_{\alpha r}(\mathcal{V} A) & =\frac{i}{2} \partial_{\alpha \dot{\alpha}} \bar{f}_{r}^{\dot{\alpha}} D_{o} A \\
\bar{D}_{\dot{\alpha}}^{r^{\prime}}(\mathcal{V} A) & =\frac{i}{2} \partial_{\alpha \dot{\alpha}} f^{\alpha r^{\prime}} D_{o}^{\prime} A
\end{aligned}
$$


Since

$$
\begin{aligned}
D_{\alpha i} K & =-\frac{i}{2} \partial_{\alpha \dot{\alpha}} \bar{f}_{i}^{\dot{\alpha}} \\
\bar{D}_{\dot{\alpha}}{ }^{i} \bar{K} & =\frac{i}{2} \partial_{\alpha \dot{\alpha}} f^{\alpha i}
\end{aligned}
$$

we see that the desired transformation rule for $A$ is

$$
\delta A=\mathcal{V} A+\left(c K-c^{\prime} \bar{K}\right) A
$$

where

$$
D_{o} A=c A ; \quad D_{o}^{\prime} A=c^{\prime} A
$$

This transformation rule can be rewritten in the form

$$
\begin{aligned}
\delta A= & f_{r}{ }^{s^{\prime}} D_{s^{\prime}}{ }^{r} A+f_{r} s^{\prime \prime} D_{s^{\prime \prime}}{ }^{r} A+f_{r^{\prime \prime}}{ }^{\prime} D_{s^{\prime}}{ }^{\prime \prime} A+f_{r^{\prime \prime}} s^{\prime \prime} \hat{D}_{s^{\prime \prime}}{ }^{\prime \prime} A \\
& +c\left(K+f_{o}-\frac{p}{r} f_{o}^{\prime \prime}\right) A-c^{\prime}\left(\bar{K}-f_{o}^{\prime}+\frac{q}{r} f_{o}^{\prime \prime}\right) A
\end{aligned}
$$

The parameters $f_{o}-\frac{p}{r} f_{o}^{\prime \prime}$ and $f_{o}^{\prime}-\frac{q}{r} f_{o}^{\prime \prime}$ are independent of the dilation and R-symmetry parameters, so that the weights for these two transformations are determined by the parameters $c$ and $c^{\prime}$. Using the fact that

$$
\begin{aligned}
D_{o} u_{r} & =\frac{N-p}{p N} u_{r} \\
D_{o} u_{r^{\prime}} & =-\frac{1}{N} u_{r^{\prime}} \\
D_{o} u_{r^{\prime \prime}} & =-\frac{1}{N} u_{r^{\prime \prime}}
\end{aligned}
$$

together with similar formulae for $D_{o}^{\prime}$ and $D_{o}^{\prime \prime}$, it is straightforward to show that

$$
c=m_{1}-\frac{m}{N}, \quad c^{\prime}=-\frac{m}{N}
$$

We therefore find

$$
L=m_{1}, \quad R=\frac{2 m}{N}-m_{1}
$$

which is the correct series $\mathrm{C}$ result.

It is straightforward to adapt the above calculation to the special cases $p=0, q=0$. For $N=4$ we have two possibilities, according to whether the superconformal group is chosen to 
be $P S U(2,2 \mid 4)$ or $S U(2,2 \mid 4)$. In both cases R-symmetry does not act on the coordinates, and in the former case it is absent. The parameter $D \cdot f+\bar{D} \cdot \bar{f}$ does act on the coordinates and is therefore not the R-parameter; it is the parameter of $U(1)_{Y}$ transformations in the terminology of [34]. Since the corresponding generator does not belong to the superconformal algebra in either case the parameter $D \cdot f+\bar{D} \cdot \bar{f}$ must be set to zero.

For $P S U(2,2 \mid 4)$ the results derived above can be straightforwardly taken over provided one sets $R=0$. In the $S U(2,2 \mid 4)$ case, things are a little more subtle. It is possible to infer the existence of a real superfield parameter $\hat{R}$, say, whose leading component is the $R$ parameter and which satisfies

$$
D_{\alpha i} \hat{R}=\frac{1}{4} \partial_{\alpha \dot{\alpha}} \bar{f}_{i}^{\dot{\alpha}}
$$

One can then define the chiral superfield

$$
K=\frac{1}{4} \partial \cdot F-i \hat{R}
$$

and proceed as before. The only difference is that the R-transformation so defined does not act on the coordinates, although it does act on the superfield itself multiplicatively.

\section{Acknowledgement}

This research was supported in part by PPARC SPG grant 613.

\section{References}

[1] M.Flato and C. Fronsdal, Lett. Math. Phys. 8 (1984) 159; V.K. Dobrev and V.B. Petkova, Phys. Lett. B162 (1985) 127, Fortschr. Phys. 35 (1987) 537; B. Binegar, Phys. Rev. D34 (1986) 525; B. Morel, A. Sciarrino and P. Sorba, Phys. Lett B166 (1986) 69, erratum B167 (1986) 486.

[2] J. Maldacena, The large $N$ limit of superconformal field theories and supergravity, Adv. Theor. Math. Phys. 2 (1998) 231-252, hep-th/9711200; S.S. Gubser, I.R. Klebanov and A.M. Polyakov, Gauge theory correlators from noncritical String theory, Phys. Lett. B428 (1998) 105, hep-th/9802109; E. Witten, Anti-de Sitter space and holography, Adv. Theor. Math. Phys. 2 (1998) 253-291, hep-th/9802150.

[3] L Andrianopoli and S Ferrara $K-K$ excitations on $A d S_{5} \times S^{5}$ and $N=4$ primary superfields, Phys. Lett. B430 (1998) 248-253.

[4] L Andrianopoli and S Ferrara On short and long $S U(2,2 \mid 4)$ multiplets in the AdS/CFT correspondence, Lett. Math. Phys. 48 (1999) 145, hep-th/9812067.

[5] S. Ferrara and A. Zapparoni, Superconformal fields, multiplet shortening and the $A d S_{5} / \mathrm{SCFT}_{4}$ correspondence hep-th/9908163 
[6] P.S. Howe and G.G. Hartwell, A superspace survey, Class. Quant. Grav. 12 (1995) 18231880.

[7] W. Siegel, Nucl. Phys. B177 (1981) 325.

[8] P.S. Howe, K.S. Stelle and P.K. Townsend, Nucl. Phys. B181 (1981) 445; Nucl. Phys. B182 (1981) 332.

[9] G.G. Hartwell and P.S. Howe $(N, p, q)$ harmonic superspace, Int J. Mod. Phys 10, (1995) 3901-3919.

[10] P.S. Howe and P.C. West, Nonperturbative Green's functions in theories with extended superconformal symmetry Int. J. Mod. Phys. A14 (1999) 2659-2674, hep-th/9509140.

[11] P.S. Howe and P.C. West, Is $N=4$ Yang-Mills soluble? (1996) in Moscow 1996 Physics, 622-626, hep-th/9611074; P.S. Howe, E. Sokatchev and P.C. West, Three-point functions in N=4 Yang-Mills, Phys. Lett. B444 (1998) 341-351, hep-th/9808162; B. Eden, P.S. Howe and P.C. West, Nilpotent invariants in N=4 SYM, Phys. Lett. B463 (1999) 19-26, hepth/9905085; B. Eden, P.S. Howe, C. Schubert, E. Sokatchev and P.C. West, Extremal correlators in four-dimensional SCFT, Phys. Lett. B472 (2000) 323-331, hep-th/9910150; B. Eden, P.S. Howe, A. Pickering, E. Sokatchev and P.C. West, Four point functions in $N=2$ superconformal field theories, hep-th/0001138, to be published in Nucl. Phys. B; B. Eden, P.S. Howe, C. Schubert, E. Sokatchev and P.C. West, Four-point functions in $N=4$ supersymmetric Yang-Mills theory at two loops, Nucl. Phys. B557 (1999) 355-379, hep-th/9811172; B. Eden, C. Schubert and E. Sokatchev, Three-loop four-point correlator in $N=4 S Y M$, LAPTH-786/2000, hep-th/0003096.

[12] M. Gunaydin and N. Marcus, Class. Quant. Grav. 2 (1985) L11; M. Gunaydin, L.J. Romans and N. Warner, Phys. Lett. 154B (1985) 268.

[13] P.S Howe, On harmonic superspace, in Supersymmetries and Quantum Symmetries, eds. J. Wess and E. Ivanov, Lecture Notes in Physics, Springer (1999) 68-78, hep-th/9812133

[14] O Aharony, M Berkooz and N Seiberg, Light-cone descritption of $(2,0)$ conformal field theories in six dimensions, Adv. Theor. Math. Phys. 2 (1998) 119-153.

[15] R G Leigh and M Rozali, The large $N$ limit of (2,0) superconformal field theory, Phys. Lett. B431 (1998) 311-316.

[16] M Gunaydin and S. Takemae, Unitary supermultiplets of $O S p(8 \mid 4)$ and AdS/CFT duality, hep-th/9910110.

[17] L. Adrianopoli, S. Ferrara, E. Sokatchev and B. Zupnik, Shortening of primary operators in N-extended SCFT and harmonic sueprspace analyticity, hep-th/9912007.

[18] S. Ferrara and E. Sokatchev, Short representations of $S U(2,2 \mid N)$ and harmonic superspace analyticity.

[19] S. Ferrara and E. Sokatchev, Representations of $(1,0)$ and $(2,0)$ superconformal algebras in six dimensions: massless and short superfields, hep-th/0001178. 
[20] S. Ferrara and E. Sokatchev Conformal primaries of $O S p(8 \mid 4, \mathbb{R})$ and BPS states in $A d S_{4}$, hep-th/0003051.

[21] G. Chalmers and K. Schalm, Holographic nomral ordering and multi-particle states in the AdS/CFT correspondence, hep-th/9901144; O. Aharony, S.S. Gubser, J. Maldacena, H. Ooguri and Y. Oz, Large $N$ fiield theories, string theory and gravity, hep-th/990511; M. Bianchi, S. Kovacs, G. Rossi and Y. Stanev, On the logarithmic behaviour in $N=4$ SYM theory, hep-th/9906188; W. Skiba, Correlators of Short Multi-Trace Operators in $N=4$ Supsymmetric Yang-Mills Theory, hep-th/9907088; E. d'Hoker, D.Z. Freedman, S.D. Mathur, A. Matusis and L. Rastelli, Extremal correlators in the Ads/CFT correspondence, hep-th/9908160; E. d'Hoker, S.D. Mathur, A. Matusis and L. Rastelli, The operator product expansion of $N=4 S Y M$ and the four-point functions of supergravity, hep-th/9911222

[22] A Galperin A, E Ivanov, S Kalitzin, V Ogievetsky and E Sokatchev, Unconstrained $N=2$ matter, Yang-Mills and supergravity theories in harmonic superspace, Class. Quantum Grav. 1 (1984) 469.

[23] A Galperin, E Ivanov, S Kalitzin, V Ogievetsky and E Sokatchev, Unconstrained off-shell $N=3$ supersymmetric Yang-Mills theory, Class. Quantum Grav. 2 (1985) 155.

[24] A Galperin A, E Ivanov, V Ogievetsky, Superspaces for $N=3$ supersymmetry, Soviet Journal for Nuclear Physics 46 (1987) 543.

[25] E. Ivanov, S. Kalitsin, Nguyen Ai Viet and V. Ogievetsky, J. Phys. A 18 (1985) 3433.

[26] A. Galperin, E. Ivanov and V. Ogievetsy, JETP Lett. 33 (1981) 176.

[27] S. Ferrara, J. Wess and B. Zumino, Phys. Lett. B51 (1974) 239.

[28] A A Rosly and A S Schwarz, Supersymmetry in spaces with auxiliary variables, Comm. Math. Phys. 105, (1986) 645.

[29] R Baston and M Eastwood, The Penrose Transform, Oxford University Press 1989.

[30] Y I Manin, Gauge Field Theory and Complex Geometry, Springer-Verlag 1988.

[31] A. Ferber, Nucl. Phys. B132 (1978) 55.

[32] M.F. Sohnius, Nucl. Phys. 136 (1978) 461.

[33] S. Deser, J.H. Kay and K.S. Stelle, Phys. Rev. Lett. 38 (1977) 527; S. Deser and J.H. Kay, Phys. Lett. 76B (1978) 400; R. Kallosh, Phys. Lett. 99B (1981) 122; P.S. Howe, K.S. Stelle and P.K. Townsend, Nucl. Phys. B191 (1981) 445.

[34] K. Intriligator, Bonus Symmetries of $\mathcal{N}=4$ Super Yang-Mills Correlation Functions via AdS Duality, hep-th/9811047. 